\title{
„Diabesity“ - Adipositas und Typ-2-Diabetes (Update 2019)
}

\author{
Hermann Toplak · Deborah Raphaela Leitner · Jürgen Harreiter · Friedrich Hoppichler · Thomas C. Wascher • \\ Karin Schindler · Bernhard Ludvik
}

Zusammenfassung Adipositas und Typ-2-Diabetes werden seit einigen Jahren vermehrt unter dem $\mathrm{Na}$ men „Diabesity“ zusammengefasst. Das trägt dem Umstand Rechnung, dass die Adipositas dem Diabetes in den meisten Fällen häufig vorangeht und wohl der wichtigste Faktor in der weltweiten Zunahme des Typ-2-Diabetes ist. Der Body-Mass-Index (BMI) ist individuell betrachtet ein sehr grobes Maß der Körperverfettung. Sogar Normalgewichtige können bei Muskelmangel zu viel Körperfett aufweisen (Sarkope-

\section{H. Toplak ( $₫)$}

Klinische Abteilung für Endokrinologie und Diabetologie, Universitätsklinik für Innere Medizin, Medizinische Universität Graz, Auenbruggerplatz 15, 8036 Graz, Österreich hermann.toplak@medunigraz.at

\section{R. Leitner}

Universitätsklinik für Innere Medizin, Medizinische Universität Graz, Graz, Österreich

\section{J. Harreiter}

Gender Medicine Unit, Klinische Abteilung für Endokrinologie und Stoffwechsel, Universitätsklinik für Innere Medizin III, Medizinische Universität Wien, Wien, Österreich

\section{F. Hoppichler}

Abteilung für Innere Medizin, Krankenhaus der Barmherzigen Brüder Salzburg, Salzburg, Österreich

T. C. Wascher

Medizinische Abteilung, Hanusch-Krankenhaus, Wien, Österreich

\section{K. Schindler}

Universitätsklinik für Innere Medizin III, Medizinische Universität Wien, Wien, Österreich

\section{B. Ludvik}

Medizinische Abteilung mit Diabetologie, Endokrinologie und Nephrologie, Krankenanstalt Rudolfstiftung, Wien, Österreich nie), weswegen zusätzlich Messungen des Bauchumfanges und des Körperfettes empfohlen werden (z. B. Bioimpedanzanalyse [BIA]). Lebensstilmanagement mit Ernährungsumstellung und Bewegung ist eine der wichtigsten Maßnahmen in der Diabetesprävention und -therapie. In der Therapie des Typ-2-Diabetes hat das Gewicht als sekundärer Zielparameter zunehmende Bedeutung erlangt. Auch die Wahl der antidiabetischen Therapie, aber auch der Begleittherapien nimmt immer mehr darauf Rücksicht. Welchen Stellenwert Antiadiposita in der antidiabetischen Therapie erlangen werden, wird durch zukünftige Studien mit Gewicht als primärem Endpunkt zu klären sein. Die bariatrische Chirurgie ist derzeit bei einem Typ2 -Diabetes mit BMI $>35 \mathrm{~kg} / \mathrm{m}^{2}$ indiziert und $\mathrm{kann} \mathrm{zu}-$ mindest teilweise zur Diabetesremission beitragen, sie muss aber in ein entsprechendes lebenslanges Betreuungskonzept eingebunden sein.

Schlüsselwörter Adipositas · Typ-2-Diabetes · Körperzusammensetzung · Ernährung · Formula-Diäten

\section{"Diabesity"-Obesity and type 2 diabetes (Update 2019)}

Summary For several years obesity and type 2 diabetes have been increasingly summarized under the name "diabesity". This is due to the fact that in most cases obesity precedes diabetes and is the most important risk factor for the worldwide increase of type 2 diabetes. The body mass index (BMI) is a very crude measure of body fatness in individuals. Even normal weight persons can have too much body fat in cases of a lack of muscle mass (sarcopenia), which is why additional measurements of waist circumference and body fatness, e.g. bioimpedance analysis (BIA), are recommended. Lifestyle management including nutrition modification and increase in physical activity are im- 
portant measures for the prevention and treatment of diabetes. Regarding the treatment of type 2 diabetes, body weight is increasingly used as a secondary target parameter. The choice of anti-diabetic treatment and also concomitant treatment is increasingly influenced by body weight. The significance of anti-obesity medications in the treatment of type 2 diabetes will have to be clarified by future studies with body weight as the primary endpoint. Bariatric surgery is at present indicated with a BMI $>35 \mathrm{~kg} / \mathrm{m}^{2}$ with concomitant risk factors, such as diabetes and can lead at least to partial diabetes remission but has to be incorporated into an appropriate lifelong care concept.

Keywords Obesity - Type 2 diabetes · Body composition · Nutrition $\cdot$ Formula diets

Die Adipositas ist auf Basis einer entsprechenden Genetik der wohl wichtigste Risikofaktor für den Typ-2Diabetes. In der EU sind $17 \%$ der Erwachsenen zwischen 20 und 74 Jahren adipös, $36 \%$ sind übergewichtig [1]. Erhöhter BMI bedeutet erhöhte Mortalität bei Männern und Frauen, wobei dies allerdings auch für Untergewicht gilt („J shaped curve“) [2]. Weltweit ist der größte Teil des Typ-2-Diabetes der Adipositas zuzuordnen, so auch in Europa [3, 4].

Diabetes und Adipositas zusammen erhöhen das Mortalitätsrisiko auf das 7-Fache [5].

Es wird häufig darüber diskutiert, ob die Adipositas eine Erkrankung für sich selbst darstellt oder einem krankhaften Zustand entspricht, eine rezente Publikation der European Association for the Study of Obesity (EASO) hat sie zum „Gateway of ill Health“, also zu einem zentralen krankheitsbestimmenden Faktor erklärt [6] und später auch eine neue Klassifikation für ICD-11 vorgeschlagen [7]. Die „Milan Declaration 2015“ der EASO hat die Adipositas nunmehr selbst als „progressive Erkrankung“ genannt und als zentrales „Tor“ zu vielen anderen Erkrankungen wie die meisten NCDs („non communicable diseases“; nicht übertragbare Erkrankungen) erklärt. So wurde die zentrale Rolle der Adipositas bei Diabetes, Hyperlipidämie und Hypertonie mit der Konsequenz erhöhter kardiovaskulärer Morbidität und Mortalität anerkannt (http://www.easo.org). Die enge Beziehung von Adipositas und Typ-2-Diabetes hat auch zur Bezeichnung „Diabesity“ geführt.

Die WHO hat die Adipositas daher zum größten globalen chronischen Gesundheitsproblem erklärt, das neuerdings die Bedeutung der Malnutrition bei Weitem übertrifft. Im Jahr 2030 könnten neueren Projektionen zufolge etwa $60 \%$ der Weltbevölkerung übergewichtig oder adipös sein [8-10].

Neben den an anderer Stelle behandelten Themen soll der Typ-2-Diabetes im Folgenden aus diesem Blickwinkel betrachtet werden.

\section{Anthropometrische Daten}

Es ist heute erwiesen, dass jeder BMI mit einem unterschiedlichen Körperfettanteil verbunden sein kann. Zwar weisen fast $100 \%$ der Personen mit einem BMI $>30 \mathrm{~kg} / \mathrm{m}^{2}$ einen hohen Fettanteil auf, aber auch immerhin noch etwa ein Drittel der Normalgewichtigen [11]. Dies ist auf den heute häufigen Verlust der Muskelmasse und der Muskelkraft mit zunehmendem Alter oder Mangel an Muskelmasse (Sarkopenie) zurückzuführen, die eine ungünstige Fett-Muskel-Relation auch bei Normal- oder Übergewicht bedeutet [12-14]. Viele der genannten Personen werden auch über den erhöhten Bauchumfang entdeckt. Besteht eine abdominelle Fettansammlung, ist das Risiko für Atherosklerose und vorzeitige Mortalität selbst bei normalem BMI erhöht. Als Grenzwerte für den Bauchumfang gelten für eine kaukasische Bevölkerung $88 \mathrm{~cm}$ für Frauen und $102 \mathrm{~cm}$ für Männer, wobei diese Werte jedoch bei älteren Personen (geringere Körpergröße, Kyphose, Skoliose) nicht anwendbar sind. Da andere ethnische Populationen (z. B. Asiaten) bei gleichem BMI eine größere Fettmasse aufweisen, werden für diese Kollektive andere Grenzwerte diskutiert [15].

Eine korrekte Untersuchung umfasst daher zusätzlich den Bauchumfang und eine geeignete Methode zur Erfassung des Körperfettes („dual energy X-ray absorptiometry“ [DEXA], BIA, BODPOD- „air displacement plethysmography“). Zwar haben Letztere auch ihre Schwächen, geben aber eine gute Orientierung und sind besonders im Verlauf eines Gewichtsmanagements von unschätzbarem Wert [16, 17]. Ein Screening auf Sarkopenie mittels eines Fragebogens SARCF kann helfen, eine Sarkopenie frühzeitig zu erkennen $[18,19]$.

\section{Implikationen für das Lebensstilmanagement}

Beim Gewichtmanagement bevorzugen in den USA und auch weltweit in allen Kulturen Frauen Ernährungsmaßnahmen und Männer Bewegungsmaßnahmen [20, 21]. Eine Zunahme der Muskelmasse und eine Verminderung der Körperfettmasse braucht in der Regel aber bei allen Menschen beides.

Die Basis jeden Lebensstilmanagements liegt in der Einleitung der Steigerung körperlicher Aktivität. Aerobe Bewegung ist zur Verminderung des Körperfettes geeignet [22]. Bei Sarkopenie ist auf eiweißreiche Ernährung in Kombination mit Muskelaufbau durch unterstützendes Krafttraining zu achten [23]. Einen besonders wichtigen Faktor stellt neben der Muskelmasse die Funktionalität dar, weswegen im Monitoring geeignete Parameter empfohlen werden (z. B. „Handgrip-Test") [24]. Insbesondere im Alter ist die körperliche Fitness von großer prognostischer Bedeutung [25, 26].

Eine erfolgreiche Gewichtsreduktion kann nur mit einer energiereduzierten Diät, die fettreduziert, aber auch kohlenhydratreduziert sein kann und am besten 
einem mediterranen Ernährungsmuster entspricht, erreicht werden [27-29]. Eine mediterrane Ernährung konnte darüber hinaus bei Menschen mit Diabetes die Notwendigkeit der Verordnung oraler Antidiabetika bei neu diagnostizierten Diabetikern reduzieren [30].

Im Fall einer fettreduzierten Diät sollte die Kohlenhydratqualität beachtet werden (bevorzugt komplexe Kohlenhydrate, möglichst wenig Mono- und Disaccharide).

Wie von Dansinger et al. [31] sehr gut gezeigt, geht es in der Praxis darum, die Patienten zu motivieren, ihre Ernährung unter Berücksichtigung der persönlichen Präferenzen zu verändern, die Energiezufuhr zu reduzieren und diese Umstellung dauerhaft beizubehalten.

In der Ernährungstherapie sind heute individuell maßgeschneiderte Kostformen, die auch persönliche Präferenzen, Abneigungen, den kulturellen und religiösen Hintergrund sowie die individuelle ökonomische Situation in Betracht ziehen, zu erstellen.

Supplemente mit definiertem Inhalt können im Ersatz einzelner oder mehrerer (meist 2) Mahlzeiten hilfreich sein („low calorie diets“) [32]. Für kurze Zeiträume können bei entsprechender Eignung der Patienten auch stark hypokalorische ketogene Kostformen („very low calorie diets [VLCDs]") eingesetzt werden, die dann meist von „low calorie diets“ mit 1- bis 2-mal tägigem Mahlzeitenersatz (LCDs) über längere Zeit gefolgt werden.

\section{Benefit von Gewichtsverlust in Diabetespräven- tion und Therapie}

Nachdem die Mehrheit der Patienten mit Typ-2-Diabetes übergewichtig oder adipös ist, stellt die Gewichtsreduktion eine wichtige therapeutische Maßnahme in der Diabetesprävention dar. Dass diese einen positiven Einfluss auf das Diabetesrisiko ausübt, konnte bereits in mehreren Studien gezeigt werden $[33,34]$. So konnte eine Diabetesreduktion von $58 \%$ in der „Finnish Diabetes Prevention Study“ bei Patienten mit Prädiabetes durch Lebensstilintervention (Ernährungs- und Bewegungskonzept) erreicht werden [33]. Ähnliche Resultate wurden auch im „Diabetes Prevention Programme“ erzielt. Hier konnte ein moderater Gewichtsverlust mittels Lebensstilintervention die Diabetesmanifestation um $58 \%$ reduzieren und damit besser als mit Metformin alleine (ohne Lebensstiländerung) [34]. Des Weiteren belegen epidemiologische Daten den Wert einer frühen Gewichtsreduktion bei Typ-2-Diabetes. Jedes Kilo Gewichtsverlust im ersten Jahr nach Manifestation war in einer Studie von Lean et al. mit einem erhöhten „Survival“ von 3 bis 4 Monaten assoziiert, $10 \mathrm{~kg}$ Gewichtsverlust mit einer Wiederherstellung der nach der Diabetesmanifestation verminderten Lebenserwartung im Ausmaß von $35 \%$ [35]. Ein geplanter moderater Gewichtsverlust von etwa $10 \mathrm{~kg}$ konnte auch in der „Cancer Prevention
Study 1“ der American Cancer Society die Mortalität von Diabetikern um etwa $25 \%$ senken [36]. Diese überaus positiven Ergebnisse wurden auch durch die Resultate der „Look AHEAD study“ belegt. Hier wurde der Gewichtsverlust allerdings auch mit Hilfe von einer Bewegungstherapie erzielt. Durch diese Maßnahmen konnte ein moderater Gewichtsverlust von 5-10 \% die Fitness, den $\mathrm{HbA}_{1 \mathrm{c}}$-Level und die kardiovaskulären Risikofaktoren verbessern. Zusätzlich dazu konnten eine Reduktion von Diabetes, Cholesterin-senkenden und Blutdruck-stabilisierenden Medikamenten nach 1 Jahr sowie eine Reduktion von Depressionssymptomen und eine Verbesserung der Schlafapnoe beobachtet werden [37-40]. Interessanterweise ist eine Gewichtsreduktion von $>5 \%$ für diese positiven Effekte vonnöten. Nachdem diese eine überaus disziplinierte Lebensstilintervention mit kalorischer Restriktion sowie regelmäßiger körperlicher Bewegung benötigt, scheint sie nicht die geeignetste Therapie für alle übergewichtigen oder adipösen Patienten zu sein.

\section{Implikationen für die Verwendung antiadipöser medikamentöser Therapien}

Nach wie vor wird die Lebensstilintervention als primäre Adipositastherapie betrachtet, welche durch die Gabe von Antiadiposita in ihrem Erfolg unterstützt werden kann.

In Europa ist der Triglyzerid-Lipase-Hemmer Orlistat seit etwa 20 Jahren am Markt, wobei eine rezeptpflichtige Formulation (Xenical ${ }^{\circledR}, 3-\mathrm{mal} 120 \mathrm{mg}$ ) und eine OTC-Formulation am Markt sind (ALLI ${ }^{\circledR}, 3-\mathrm{mal}$ $60 \mathrm{mg}$ ). Orlistat soll gleichzeitig mit einer fettarmen Diät eingesetzt werden (die selbst eine Lebensstiltherapie mit Gewichtsreduktion unterstützt) und reduziert die Fettresorption um weitere $30 \%$, wodurch der Summeneffekt entsteht. Potenzielle Nebenwirkungen sind in erster Linie gastrointestinaler Natur (Fettstühle, Diarrhöen), insbesondere wenn eine fettreiche Ernährung als Basis genutzt wird.

Die weiteren Antiadipositamöglichkeiten variieren weltweit. Derzeit sind in den USA mehrere dieser Medikamente registriert worden, von denen Liraglutid (bis 1,8 mg bei Typ-2-Diabetes und $3 \mathrm{mg}$ bei Adipositas) und die Fix-Kombination von Naltrexon und Bupropion auch in Europa von der EMA zugelassen worden sind [41]. Beide Pharmaka sind seit 2018 auch in Österreich zur Verwendung in der Adipositastherapie erhältlich. Damit ist es nun möglich, Patienten mit Typ-2-Diabetes nicht nur hinsichtlich ihrer Gewichtsreduktion zu unterstützen, sondern gleichzeitig auch ihren $\mathrm{HbA}_{1 \mathrm{c}}$-Wert $\mathrm{zu}$ verbessern $[42,43]$. Durch diese kombinierte Betrachtung von glykämischer Kontrolle und Körpergewicht (bzw. Muskel- und Fettmasse und deren Relation) eröffnen sich somit völlig neue Strategien in der Diabetestherapie. Antiadiposita könnten die Möglichkeit mit sich bringen, Übergewicht und schlechte glykämische Kontrolle simultan zu behan- 
deln. Ob solche therapeutische Maßnahmen auch in der Zukunft empfohlen werden können, müssen zukünftige Studien klären, in welchen die Gewichtsreduktion als primäres Endziel bei Patienten mit Typ2-Diabetes untersucht wird. Derzeit können hierzu noch keine evidenzbasierten Empfehlungen abgegeben werden.

\section{Gewichtszunahme aufgrund von Begleit- therapien}

Aus Sicht der Adipositas ist auch darauf zu achten, gewichtssteigernde Begleittherapien zu vermeiden. Dies gestaltet sich jedoch meist schwierig, da sehr viele Arzneimittelgruppen ein gewichtssteigerndes Potenzial aufweisen. $\mathrm{Zu}$ diesen sind $\mathrm{u}$. a. auch viele traditionelle Antidiabetika zu rechnen. Im Allgemeinen sind heute jene antidiabetischen Therapien $\mathrm{zu}$ bevorzugen, die das Gewicht der Diabetiker nicht steigern, sondern - wenn möglich - eine Gewichtsreduktion unterstützen (Metformin, DPP-IV-Inhibitoren, SGLTInhibitoren und GLP1-Analoga) [44].

Generell gibt es einige gewichtssteigernde Begleittherapien, auf welche - wenn möglich - verzichtet oder welche durch gewichtsneutrale oder -reduzierende Alternativen ersetzt werden sollen. Hierzu zählen u. a. viele Psychopharmaka, welche zugleich auch die wichtigste gewichtssteigernde Begleittherapie per se darstellen. Des Weiteren sind auch Steroidhormone oder Betablocker für eine moderate Gewichtssteigerung bekannt [44]. Unter Letzteren ist zudem eine gehäufte Diabetesmanifestation zu beobachten [45].

\section{Bariatrische Chirurgie}

Bariatrische Operationen sind momentan die effektivste Strategie für eine langfristige Gewichtsreduktion. Generell kann dadurch eine 15 - bis $40 \%$-Reduktion des Ausgangsgewichtes erreicht werden [46], wodurch sich Adipositas-assoziierte Komorbiditäten wie Diabetes verbessern und die Gesamtmortalität reduziert wird [47-49]. Derzeit ist die Indikation für bariatrische Operationen bei Patienten mit Adipositas ohne Diabetes bei einem BMI $>40 \mathrm{~kg} / \mathrm{m}^{2}$ und bei Patienten mit Typ-2-Diabetes bei einem BMI $>35 \mathrm{~kg} / \mathrm{m}^{2}$ gegeben. Aufgrund der positiven Effekte werden bariatrische Operationen in manchen chirurgischen Zentren bereits bei viel niedrigerem BMI durchgeführt. Hierfür sind nach wie vor sorgfältige Nutzen-RisikoAnalysen aus kontrollierten Studien abzuwarten. Auch wenn dadurch die bariatrische Chirurgie einer immer größeren Patientenpopulation zugänglich gemacht wird, sollte die Patientenwahl nach wie vor äußerst gewissenhaft erfolgen, um jenen Menschen die erforderlichen Basis- und Kontrolluntersuchungen vor und nach bariatrischen Operationen zu ermöglichen. Neben der Suche nach Mangelerscheinungen sollten auf jeden Fall die Körperzusammensetzung (MuskelFett-Relation) und auch die Knochendichte - erstmals spätestens 2 Jahre nach der Operation - untersucht werden [50]. Da die multidisziplinäre medizinische Nachbetreuung dieser Patienten sehr umfangreich ist, wurden erst kürzlich dazu 2 Übersichtsarbeiten zu den Richtlinien publiziert [51, 52].

In letzter Zeit wurde die bariatrische Chirurgie auch immer wieder als mögliche Therapieoption für Typ2-Diabetes betrachtet, da sie sowohl $\mathrm{zu}$ einer signifikanten Verbesserung der Hyperglykämie beitragen als auch zu einer kompletten Diabetesremission führen kann [46, 53]. Daher wird sie in diesem Zusammenhang auch immer öfter als „metabolische Chirurgie“ bezeichnet und als Behandlungsmöglichkeit für Typ2-Diabetes empfohlen [54, 55]. Generell beträgt die Diabetesremissionsrate 45-95\%, je nachdem, welche Operation durchgeführt wurde - wobei ein größerer Gewichtsverlust zu einer höheren Diabetesremission beiträgt [56-58]. Im direkten Vergleich konnte gezeigt werden, dass bariatrische Operationen mit antidiabetischer Therapie einer rein medikamentösen Therapie hinsichtlich der Gewichtsreduktion, der glykämischen Kontrolle und dem Gebrauch von Blutzucker-regulierenden, Blutdruck-senkenden und Cholesterin-senkenden Medikamenten überlegen sind. Die Daten des erst kürzlich erschienen 5-Jahres-Follow-up-Berichtes von „STAMPEDE“ (Surgical Treatment and Medications Potentially Eradicate Diabetes Efficiently) konnten zudem zeigen, dass die positiven Effekte auch noch 5 Jahre nach der Operation anhielten [59]. Dennoch sollten bariatrische Operationen in ihrem Erfolg nicht überschätzt werden („Heilung des Diabetes“), da auch sie eine neuerliche Manifestation des Diabetes oder eine Verschlechterung desselben nach Jahren nicht ausschließen können [46]. Daher und auch wegen der möglichen Spätfolgen der Operationen sind weitere Langzeitstudien vonnöten, welche sich mit diesen Fragestellungen auseinandersetzen.

Funding Open access funding provided by Medical University of Graz.

Interessenkonflikt $\mathrm{H}$. Toplak hat von folgenden Unternehmen, Forschungsunterstützungen und/oder Honorare erhalten: Almased, Bodymed, Cheplapharm, Eli Lilly, Insumed, MSD, Mylan, Novartis, Novo Nordisk, Pfizer, Sanofi-Aventis, Servier, Omron, Deutschland, Takeda, Vivus. D. R. Leitner erhielt Beratungshonorare von Novo Nordisk. J. Harreiter hat von folgenden Unternehmen Forschungsunterstützungen und/oder Honorare erhalten: AstraZeneca, Novo Nordisk und Takeda. F. Hoppichler hat von folgenden Unternehmen Forschungsunterstützungen und/oder Honorare erhalten: Novo Nordisk, Sanofi-Aventis. T. Wascher und K Schindler geben an, dass kein Interessenkonflikt besteht. B. Ludvik hat von folgenden Unternehmen Forschungsunterstützungen und/oder Honorare erhalten: Aengus, Novo Nordisk, Sanofi-Aventis, Boehringer Ingelheim, Eli Lilly, Roche, Novartis, Takeda und Amgen.

Open Access Dieser Artikel wird unter der Creative Commons Namensnennung 4.0 International Lizenz (http:// creativecommons.org/licenses/by/4.0/deed.de) veröffentlicht, welche die Nutzung, Vervielfältigung, Bearbeitung, Verbreitung und Wiedergabe in jeglichem Medium und Format 
erlaubt, sofern Sie den/die ursprünglichen Autor(en) und die Quelle ordnungsgemäß nennen, einen Link zur Creative Commons Lizenz beifügen und angeben, ob Änderungen vorgenommen wurden.

\section{Literatur}

1. European Health Interview Survey. EUROSTAT 2016. 203/2016.2016.http://ec.europa.eu/eurostat/documents/ 2995521/7700898/3-20102016-BP-EN.pdf/c26b037b-d5f34c05-89c1-00bf0b98d646. Zugegriffen: 18. Juni 2018.

2. Prospective Studies Collaboration, et al. Body-mass index and cause-specific mortality in 900000 adults: collaborative analyses of 57 prospective studies. Lancet. 2009;373(9669):1083-96.

3. Hossain P, Kawar B, El Nahas M. Obesity and diabetes in the developing world-a growing challenge. N Engl J Med. 2007;356(3):213-5.

4. International Diabetes Federation Diabetes Atlas. 2nd ed. Brussels, International Diabetes Federation. 2003. http://www.diabetesatlas.org/component/attachments/? task=download\&id=73.Zugegriffen:28. Sept. 2017.

5. Oldridge NB, et al. Prevalence and outcomes of comorbid metabolic and cardiovascular conditions in middle- and older-age adults. JClin Epidemiol. 2001;54(9):928-34.

6. Frühbeck G, et al. Obesity: the gateway to ill health-an EASO position statement on a rising public health, clinical and scientific challenge in Europe. Obes Facts. 2013;6(2):117-20.

7. Hebebrand J, et al. A proposal of the European Association for the Study of Obesity to improve the ICD-11 diagnostic criteria for obesity based on the three dimensions etiology, degree of adiposity and health risk. Obes Facts. 2017;10(4):284-307.

8. Finucane MM, et al. National, regional, and global trends in body-mass index since 1980: systematic analysis of health examination surveys and epidemiological studies with 960 country-years and 9.1 million participants. Lancet. 2011;377(9765):557-67.

9. Kelly T, et al. Global burden of obesity in 2005 and projections to 2030. Int J Obes (Lond). 2008;32(9):1431-7.

10. WHO. Obesity and overweight fact sheet no.311. 2016. http://www.who.int/mediacentre/factsheets/fs311/en/. Zugegriffen:28. Sept. 2017.

11. Gomez-Ambrosi J, et al. Body mass index classification misses subjects with increased cardiometabolic risk factors related to elevated adiposity. Int J Obes (Lond). 2012;36(2):286-94.

12. Cruz-Jentoft AJ, et al. Sarcopenia: European consensus on definition and diagnosis: report of the European Working Group on Sarcopenia in Older People. Age Ageing. 2010;39(4):412-23.

13. Delmonico MJ, et al. Alternative definitions of sarcopenia, lower extremity performance, and functional impairment with aging in older men and women. J Am Geriatr Soc. 2007;55(5):769-74.

14. Goodpaster BH, et al. The loss of skeletal muscle strength, mass, and quality in older adults: the health, aging and body composition study. J Gerontol A Biol Sci Med Sci. 2006;61(10):1059-64.

15. WHO Expert Consultation. Appropriate body-mass index for Asian populations and its implications for policy and intervention strategies. Lancet. 2004;363(9403):157-63.

16. Tsigos C, et al. Management of obesity in adults: European clinical practice guidelines. Obes Facts. 2008;1(2):106-16.
17. Yumuk V, et al. An EASO position statement on multidisciplinary obesity management in adults. Obes Facts. 2014;7(2):96-101.

18. MorleyJE, Anker SD, von Haehling S. Prevalence, incidence, and clinical impact of sarcopenia: facts, numbers, and epidemiology-update 2014. J Cachexia Sarcopenia Muscle. 2014;5(4):253-9.

19. Malmstrom TK, et al. SARC-F: a symptom score to predict persons with sarcopenia at risk for poor functional outcomes. J Cachexia Sarcopenia Muscle. 2016;7(1):28-36.

20. Tsai SA, et al. Gender differences in weight-related attitudes and behaviors among overweight and obese adults in the United States. Am J Mens Health. 2016;10(5):389-98.

21. Grebitus C, Hartmann M, Reynolds N. Global obesity study on drivers for weight reduction strategies. Obes Facts. 2015;8(1):77-86.

22. Yumuk V, et al. European guidelines for obesity management in adults. Obes Facts. 2015;8(6):402-24.

23. Wycherley TP, et al. Long-term effects of a very low-carbohydrate weight loss diet on exercise capacity and tolerance in overweight and obese adults. J Am Coll Nutr. 2014;33(4):267-73.

24. Otto M, et al. Handgrip strength as a predictor for post bariatric bodycomposition. Obes Surg. 2014;24(12):2082-8.

25. HainerV,ToplakH, Stich V.Fator fit: what is moreimportant? Diabetes Care. 2009;32(Suppl2):S392-S7.

26. Cawthorn P, Fox KM, Gandra SR, Delmonico MJ, Chiou CF, Anthony MS, Sewall A, Goodpaster B, Satterfield S, Cummings SR, Harris TB, Health, Aging and Body Composition Study. Do muscle mass, muscle density, strength, and physical function similarly influence risk of hospitalisation in older adults? JAm Geriatr Soc. 2009;57(8):1411-9.

27. LarsenRN, etal.Theeffectofhigh-protein,low-carbohydrate diets in the treatment of type 2 diabetes: a 12 month randomised controlled trial. Diabetologia. 2011;54(4):731-40.

28. Shai I, et al. Weight loss with a low-carbohydrate, mediterranean, or low-fat diet. NEngl J Med. 2008;359(3):229-41.

29. Krebs JD, et al. The Diabetes Excess Weight Loss (DEWL) Trial: a randomised controlled trial of high-protein versus high-carbohydrate diets over 2 years in type 2 diabetes. Diabetologia.2012;55(4):905-14.

30. Esposito K, et al. Effects of a Mediterranean-style diet on the need for antihyperglycemic drug therapy in patients with newly diagnosed type 2 diabetes: a randomized trial. Ann Intern Med. 2009;151(5):306-14.

31. Dansinger ML, et al. Comparison of the Atkins, Ornish, Weight Watchers, and Zone diets for weight loss and heart disease risk reduction: a randomized trial. JAMA. 2005;293(1):43-53.

32. Wadden TA, et al. One-year weight losses in the Look AHEAD study: factors associated with success. Obesity (Silver Spring). 2009;17(4):713-22.

33. Lindström J, et al. The Finnish Diabetes Prevention Study (DPS): lifestyle intervention and 3-year results on diet and physical activity. Diabetes Care. 2003;26(12):3230-6.

34. Knowler WC, et al. Reduction in the incidence of type 2 diabetes with lifestyle intervention or metformin. N Engl J Med.2002;346(6):393-403.

35. Lean ME, et al. Obesity, weight loss and prognosis in type 2 diabetes. Diabet Med. 1990;7(3):228-33.

36. Williamson DF, et al. Intentional weight loss and mortality among overweight individuals with diabetes. Diabetes Care. 2000;23(10):1499-504.

37. Wing RR, et al. Benefits of modest weight loss in improving cardiovascular risk factors in overweight and obese individuals with type 2 diabetes. Diabetes Care. 2011;34(7):1481-6. 
38. LookARG, WingRR.Long-termeffects ofalifestyleintervention on weight and cardiovascular risk factors in individuals with type 2 diabetes mellitus: four-year results of the Look AHEAD trial. Arch Intern Med. 2010;170(17):1566-75.

39. Foster GD, et al. A randomized study on the effect of weight loss on obstructive sleep apnea among obese patients with type 2 diabetes: the Sleep AHEAD study. Arch Intern Med. 2009;169(17):1619-26.

40. Rubin RR, et al. Impact of intensive lifestyle intervention on depression and health-related quality of life in type 2 diabetes: the Look AHEAD Trial. Diabetes Care. 2014;37(6):1544-53.

41. Toplak H, et al. 2014 EASO position statement on the use of anti-obesity drugs. Obes Facts. 2015;8(3):166-74.

42. Berne C, Orlistat Swedish Type 2 diabetes Study. A randomized study of orlistat in combination with a weight management programme in obese patients with Type 2 diabetes treated with metformin. Diabet Med. 2005;22(5):612-8.

43. Hollander P, et al. Effects of naltrexone sustained-release/bupropion sustained-release combination therapy on body weight and glycemic parameters in overweight and obese patients with type 2 diabetes. Diabetes Care. 2013;36(12):4022-9.

44. Leitner DR, et al. Obesity and type 2 diabetes: two diseases with a need for combined treatment strategies-EASO Can lead the way. Obes Facts. 2017;10(5):483-92.

45. Zidek W, et al. First-line antihypertensive treatment in patients with pre-diabetes: rationale, design and baseline results of the ADaPT investigation. Cardiovasc Diabetol. 2008;7:22.

46. Sjöström L, et al. Lifestyle, diabetes, and cardiovascular risk factors 10 years after bariatric surgery. N Engl J Med. 2004;351(26):2683-93.

47. Pories WJ. Bariatric surgery: risks and rewards. J Clin Endocrinol Metab. 2008;93(11 Suppl 1):S89-S96.

48. SjöströmL. Review of thekeyresultsfrom theSwedish Obese Subjects (SOS) trial-a prospective controlled intervention study of bariatric surgery. J Intern Med.2013;273(3):219-34.
49. Neovius M, et al. Health care use during 20 years following bariatric surgery. JAMA. 2012;308(11):1132-41.

50 . Thibault $\mathrm{R}$, et al. Twelve key nutritional issues in bariatric surgery. Clin Nutr. 2016;35(1):12-7.

51. Busetto L, et al. Practical recommendations of the obesity management task force of the European association for the study of obesity for the post-bariatric surgery medical management. Obes Facts. 2017;10(6):597-632.

52. Busetto L, Dicker D, Azran C, Batterham RL, FarpourLambert N, Fried M, et al. Obesity management taskforce of the European Association for the Study of Obesity Released "practical recommendations for the post-bariatric surgery medical management”. Obes Surg. 2018;28(7):2117-2121. https://doi.org/10.1007/s11695-018-3283-z

53. Sjöholm K, et al. Incidence and remission of type 2 diabetes in relation to degree of obesity at baseline and 2 year weight change: the Swedish Obese Subjects (SOS) study. Diabetologia.2015;58(7):1448-53.

54. Rubino F, etal. Metabolic surgery in the treatment algorithm for type 2 diabetes: a joint statement by international diabetes organizations. Diabetes Care. 2016;39(6):861-77.

55. Schauer PR, Nor Hanipah Z, Rubino F. Metabolic surgery for treating type 2 diabetes mellitus: Now supported by the world's leading diabetes organizations. Cleve Clin J Med. 2017;84(7 Suppl 1):S47-S56.

56 . Dixon JB, et al. Surgery as an effective early intervention for diabesity: why the reluctance? Diabetes Care. 2005;28(2):472-4.

57. Buchwald $\mathrm{H}$, et al. Weight and type 2 diabetes after bariatric surgery: systematic review and meta-analysis. Am J Med. 2009;122(3):248-256e5.

58. Buchwald $\mathrm{H}$, etal. Bariatric surgery: a systematic review and meta-analysis. JAMA. 2004;292(14):1724-37.

59. Schauer PR, et al. Bariatric Surgeryversus Intensive Medical Therapy for Diabetes-5-Year Outcomes. N Engl J Med. 2017;376(7):641-51. 